\title{
DIFFERENT TYPES OF GRAPHS TO MODEL A CITY
}

\author{
TARAS AGRYZKOV, JOSE LUIS OLIVER, LEANDRO TORTOSA \& JOSE FRANCISCO VICENT \\ University of Alicante, Spain
}

\begin{abstract}
A city may be represented by spatial networks. The mathematical structure that defines a spatial network is a graph, which is constructed from a set of nodes and a set of edges. According to the meaning we assign to nodes and edges, different types of graphs describing urban networks may be obtained, each of them with their special characteristics. In this paper, we study five different types of graphs associated to the spatial network of the urban layout. They are briefly described and compared pointing out their main features. A case study is performed using the urban network of Alicante, Spain. By means of the study of the distribution of degrees of each one of the graphs obtained experimentally certain conclusions are obtained about the morphology of the urban network itself.

Keywords: graphs, spatial networks, urban studies, degree distribution, Delaunay triangulation.
\end{abstract}

\section{INTRODUCTION}

The city is a dynamic reality, the most complex product manufactured by a human being, which gives a shape to a society, information, space and time [1], [2]. It is worth noting, that according to reports of United Nations, one-half of people living in the world reside, work, and in general, interact with each other in the urban space. This remarkable urban growth and sprawl largely relies on economic development and constant migration of people from the outlying and rural areas for seeking the better service facilities, job and education opportunities [3]. Each time cities are getting bigger and charge a higher impact on an economic and social development of nations [4]. As we can see, there are enough reasons to be a city an attractive subject of study for numerous researchers and research groups. The study of a city encourages a multidisciplinary approach, especially with the latest technological revolutions and digitalization of a physical space of the last two decades. The city become a real laboratory to join and implement different theories from diverse areas of study: sociology, biology, computer science, mathematics and urban design among others.

It is a fact that contemporary city is a huge data source and therefore information [5]. The data from urban environment depicts different types of places, containers and contents, as well as an activity of its users. Today, with the aid of Internet, city users provide its customized geo-referenced information, contributing the global information through widely diffused mobile technologies, such as mobile phones, laptops and tablets. By sharing its everyday experience through different Internet applications, people provides an additional digitalized layer of knowledge on how they perceive and use the urban space [6], [7].

In order to analyse the complexity of a city we need to develop characteristic model capable to summarize all that data related to the physical material layer and the immaterial digitalized layer of a city. The main propose of such a model is to simplify the complexity of an urban system by impinging on those most relevant to study specific aspects. The most suitable model for urban space discretization is a spatial network [8]-[10]. This kind of model for spatial simplification represents the topology of the urban layout. Moreover, spatial network serves as a data structure that allows us both store and relate geospatial data.

Spatial networks or also called street networks can be represented as a graph, which is a mathematical data structure defined by two not empty sets: a set of nodes and a set of 
edges. Once the city is represented as a graph we can study its different topological properties and also a set of so called centralities [9], [11]. Centrality is one of the most studied concepts in the network analysis. Up to now, numerous measures have been developed, including classical measures as degree centrality, closeness, betweenness and its variants [12], [13], eigenvector centrality [14], information centrality, PageRank centrality, flow centrality [15], and many others. This set of centrality measures together with the information associated to the network allow us to better understand the behaviour of the complex system as it is the city.

In order to represent the urban space through a graph we can take different approaches, that is to say, there are many different types of graphs that are suitable for defining the urban plot system. Each of these graphs has its own set of characteristics and particularities. The main objective of this work is to review and compare different models of graphs for urban layout representation.

This paper has been divided into two parts. The first part of this paper is focused on examining the most commonly used models of spatial networks along with their essential characteristics. While, the second part of this paper presents the application of some of these spatial networks to the city of Alicante. From the urban layout of Alicante represented through different formats of spatial network, the characteristics of each of the formats are studied and compared.

\section{PRELIMINARIES}

In this section, we briefly introduce the basic concepts and properties of the graph theory that will be necessary to understand the content of this paper.

The spatial network can be represented as a planar undirected graph. Let us consider in the following a planar undirected graph $G(V, E)$, a mathematical entity where $V$ is a set of $n$ vertices or nodes $v_{1}, v_{2}, \ldots, v_{n}$ connected through a set $E$ of $m$ edges $e_{1}, e_{2}, \ldots, e_{m}$.

Associated to a graph $G$, we have some different matrices; the commonly used are adjacency, incidence and degree. We briefly describe them as they will be used in the following sections.

The adjacency matrix $A(G)=\left(a_{i j}\right)_{i, j=1}^{n}$ of the graph $G$ is a square $n \times n$ matrix such that its element $a_{i j}$ is 1 if there is an edge from vertex $i$ to vertex $j$, and 0 when there is no edge, with the diagonal elements of the matrix all 0 .

The incidence matrix $B(G)=\left(b_{i j}\right)$, for $i=1,2, \ldots, n$ and $j=1,2, \ldots, m$ of the graph $G$ is $n \times m$ matrix such that its element $b_{i j}$ is 1 if the edge $e_{j}$ and the vertex $v_{i}$ are incident and 0 otherwise.

Before we define the degree matrix, let us define the concept of degree of the node. The degree $k_{i}$ of a node $v_{i}$ is the number of edges incident to the node and defined as $k_{i}=\sum_{j=1}^{n} a_{i j}=\sum_{j=1}^{m} b_{i j}$. While the average degree of the graph is calculated as $\widehat{k}=$ $1 / n \sum_{i=1}^{n} k_{i}=2 m / n$. The way the degree is distributed among nodes of the network is an important concept, which can be calculated by the degree distribution $P(k)$, the probability to find the node with the degree $k$. The degree distribution is defined as $P(k)=n(k) / n$, where $n(k)$ is the number of nodes with $k$ edges. In general terms, this measure represents the heterogeneity of networks.

The degree matrix $D(G)=\left(d_{i i}\right)$ for $i=1,2, \ldots, n$, is a diagonal matrix whose elements are the degree of the nodes. 


\section{DIFFERENT GRAPH MODELS TO REPRESENT SPATIAL NETWORKS}

The purpose of this section is to review different graph models that are frequently used to represent spatial networks. We begin with the basic graph named primal graph, which has a direct relationship with the geometry and the topology of the urban layout.

\subsection{Primal graph}

The earliest model of spatial network is defined as planar undirected graph, proposed by Leonard Euler in 1659. This definition of spatial network reinterprets an urban space preserving correspondence between dimensions of physical entities and dimensions of abstract entities of the graph. That is to say, zero-dimensional urban entities such as street intersections are interpreted as zero-dimensional graph entities (nodes) and onedimensional urban entities such as streets are turned into one-dimensional graph entities (edges). Because of this direct correspondence between physical entities and abstract entities, this form of representation of the urban space is defined as primal graph representation [16]. Fig. 1 shows an example of the abstract urban space (left) and its corresponding primal graph (right).

The primal graph $G$ can be described by the adjacency matrix $A(G)$, which can be obtained from the incidence matrix $B(G)$ and the degree matrix $D(G)$ by the following expression:

$$
A(G)=B(G) B(G)^{T}-D(G)
$$

It is a fact that the urban layout can be discretized by primal graph as it has been shown from the beginning of the graph theory. As a primal graph, it is a planar graph, it exhibits a linear relationship between its vertices, edges and faces. This characteristic is an important issue, since it allows direct interpretation and calculation without additional computational cost of the basic graph properties. The main advantage of the primal graph is that it captures both, the geometric and topological properties of the urban fabric. This characteristic makes this format of spatial network representation very popular among different geo-spatial data bases to define transport networks and pedestrian paths.

It is worth noting that the entities of the primal graph can be used as information terminals. We can assign any king of geo-spatial data to both its vertices and edges. This feature converts the primal graph into a nonlinear data structure which relate the information of the city according to the topology of its urban layout.
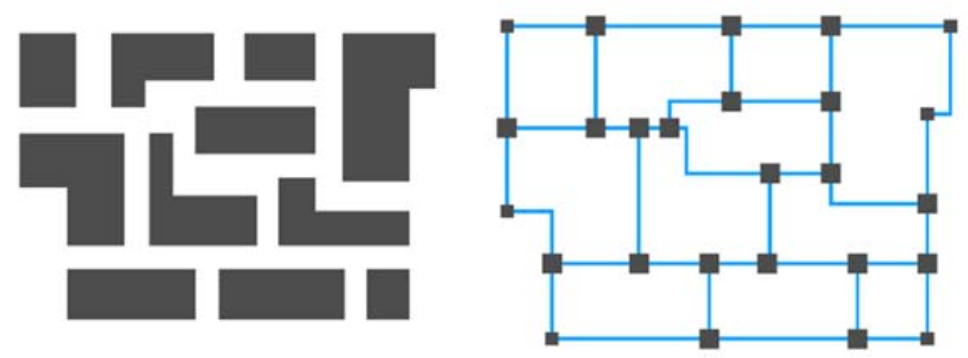

Figure 1: An example of the abstract urban space (left) and its corresponding primal graph (right). 
Spatial network defined by the primal graph belong to a particular type of complex networks. They do not belong to the scale-fee networks [17] since its degree distribution $P(k)$ does not follow the power low, given by expression $P(k) \sim c k^{-\alpha}$. Also, they do not exhibit small-world properties [18] as they are planar and there can be large topological distances between some nodes. This type of spatial networks is characterized by their low and uniform degree distribution, an issue that makes them not appropriate to be applied directly the theory of complex networks. In order to study urban networks under the prism of complex networks we can take two complementary approaches. The first one, consists on counteract the primal graph degree shortage with an additional information which can be related to any properties or additional data of the urban context. The second approach is to redefine the urban network by using another model of spatial discretization, such as the dual of primal graph, which constitutes the objective of the next section.

\subsection{Dual graph and its variants}

The dual graph approach is another model to represent spatial networks [19]. The duality, in general terms, can be defined as translation from one concept into another in one to one fashion by an involution operation. In this sense, the undirected edge to vertex dual graph or also called line graph [20] is obtained by dimension reversing duality. We translate the one-dimensional entities of the primal graph into zero-dimensional entities of the dual graph and zero-dimensional entities of the primal graph into one-dimensional entities of the dual graph. That is to say, edges of the primal graph become nodes of the dual graph and vice versa, nodes of the primal graph become edges of the dual graph. In Fig. 2 we show the street axis of an abstract urban space (right) and the edge to vertex dual graph (right).

The adjacency matrix $A\left(G_{E}\right)=\left\{a_{i j}\right\}^{m}$ of the undirected dual graph $G_{E}$ can be directly obtained from the incidence matrix $B(G)$ by the expression

$$
A\left(G_{E}\right)=B(G)^{T} B(G)-2 I_{m}
$$

The matrix $A\left(G_{E}\right)$ is $m \times m$ square matrix, which describes the linkage of a set of $m$ nodes through a set of $n$ edges.

The direct usage of edge to vertex dual graph to represent the urban network still gives us a simple structure of the network. The simplicity of the structure can be verified through its fairly uniform degree distribution. This means that by applying the complex network theory we are not able to obtain the relevant information about morphological properties of
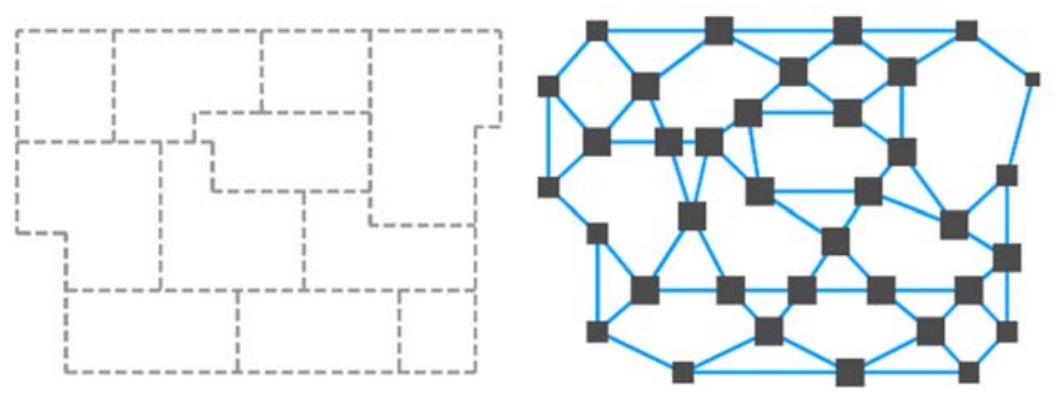

Figure 2: Street Axes of an abstract urban space (left) and edge to vertex dual graph(right). 
the urban network. In order to discover significant properties of the urban network morphology we have to adopt other specific criteria, such as criteria of continuity for edge definition. This criterion consists on merging meaningless individual edges of the graph into meaningful elements of the urban space. There are at least three different approaches for merging primal graph edges or dual graph nodes into meaningful elements of the urban environment that have been adopted in the literature.

The first approach is so called "named-street approach" [21], where the principle of continuity for edge definition is followed the criteria of street names. That is to say, each node of the graph represents a portion of the urban space associated to a specific street name.

It is a valid criterion and it is occasionally used for urban network definition. It is being found that the general tendency of degree distribution of the urban network configured by using the named-street approach follows the power low, and a small-world character for large street networks [21]. Here we have to highlight that this criterion has some important drawbacks [19], [22]. The main drawback of this criteria is that the whole process of edges merging relies on a very accurate information about street names. Unfortunately, there is no database that guarantees that all streets have valid names or, in general, all streets of a city even have the referencing name. In addition, we have to take into account that street names do not always follow the geometric or topological properties of the urban layout, they are merely labels for geographical referencing that limit the urban space following, in some cases, the very subjective criteria.

Another criterion of continuity for edge definition is the linearity of the street space. It is based on perceptual grouping, which is an important concept of Gestalt psychology [23]. This concept has also applications in human and computer vision for pattern recognition. In this case, following this criterion we merge primal graph edges which have an obtuse angle in between [24]. The purpose of this criteria is to extend the identity of an urban street over a plurality of graph edges. Following this criterion each node of the dual graph becomes to represent a straight portion of an urban space, as shown in the example in Fig. 3.

By using this approach, we can gain significantly the increase of node degrees. Furthermore, the irregular street definition involves breaking the uniformity of degree distribution. It is being shown that following this criterion, the degree distribution $P(k)$, in most cases, follows a power law [22], which is the main characteristic of scale-free networks.

The generalization of this criteria to define street networks has been proposed by Hiller [25], [26] and its colleagues from the Space Syntax Laboratory. Its theory states that an urban structure is composed of two things: a large collection of buildings linked by space, which is a physical part of the city and a complex system of human activity linked by

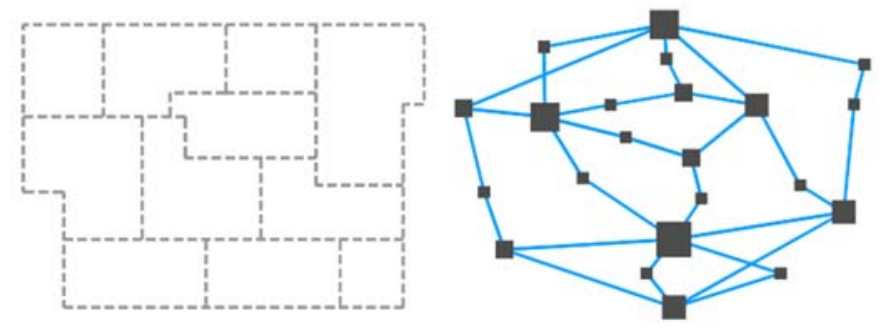

Figure 3: Street Axes of an abstract urban space (left) and edge to vertex dual graph following the linearity of street criteria (right). 
interactions, which represents a social part of a city. Following this theory, Space Syntax defines the model of axil map to quantify and compare accessibility patterns of an urban space. The axial map uses the criteria of linearity of the urban space, it is defined as a minimal set of straight lines of mayor length that represent unobstructed pedestrian movement through open spaces of the urban layout [26]. By decomposing a space in this manner, we can create a graph where straight lines represent graph nodes and its intersections become edges of the graph. The Fig. 4 shows the example of axial maps of an abstract urban space.

There are other criteria to discretize the space through the edge to vertex dual graph, such as "Intersection Continuity Negotiation", which extend the criteria of linearity of streets to define a more generic node [19].

The duality of primal graph may be related to another component of the planar graph, different from edges, such as the graph faces [27]. In this case, we translate the twodimensional entities of the primal graph into zero-dimensional entities of the dual graph and one-dimensional entities of the primal graph into one-dimensional entities of the dual graph. That is to say, faces of the primal graph become nodes of the dual graph and edges, joining faces of the primal graph, become edges of the dual graph. In Fig. 5 it is shown an example of the dual graph by using faces of the primal graph.

This model for urban network definition is strongly influenced by topology of primal graph. In this case, the necessary condition for the correct dual graph definition requires that the smallest degree of primal graph nodes must satisfy $k \geq 2$. This model is very useful, especially if we want to relate blocks or regions of the built space of the city. Note, that the result dual graph completely omits the transitional urban space of the city, it is only focused on representing the topological relationship of the built regions of an urban environment.

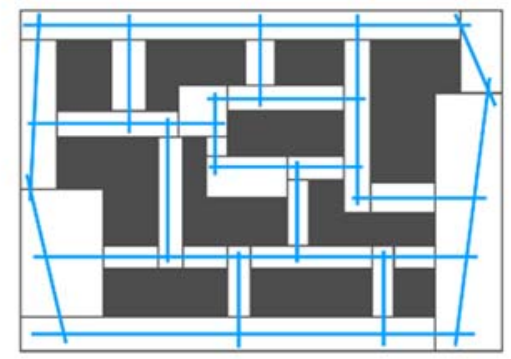

Figure 4: Axial maps of an abstract urban space.

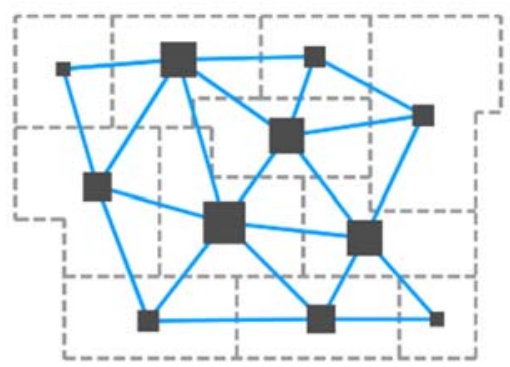

Figure 5: Face to vertex dual graph of an abstract urban space. 
The main advantage of this model for spatial discretization, is that it allows a direct and precise assignation of data, representing the attributes of the built space, to the nodes of the graph. This feature provides a consistent model to organize the city data following the topology of the urban layout.

\subsection{Delaunay graph}

The model of spatial network that we present in this section has a different propose than those we have studied in the previous sections. The main objective of this model is not to define the topology of the urban layout, but to establish the spatial relationship between the components of the urban environment. The model that best fits these requirements is the proximity graph [28] which connects a pair of nodes if and only if the nodes satisfy specific geometric requirements. The Euclidian distance is commonly used for this geometric requirement. In this case, Delaunay triangulation [29] is clear example of a proximity graph, which connects in an optimal way the nodes following the nearest-neighbour criteria. The optimal connections are verified from its dual which is the diagram of Voronoi.

Delaunay graph is a planar graph which have $m-n+1$ faces, and where each face is formed by exactly three vertices. The degree distribution of this graph depends solely on the spatial arrangement of the nodes, where each node has the degree $k \geq 2$. It is important to highlight that Delaunay graph can be used as the starting point to obtain another type of commonly used proximity graph called Gabriel graph [30], which is a subgraph of the Delaunay triangulation.

In the context of the spatial networks the Delaunay graph model can be applied to different entities of the primary graph such as their faces or their nodes. Therefore, the nodes of the Delaunay graph may represent both the intersections of the urban streets and the built urban space which is not transited by pedestrian or vehicle space. The Fig. 6 represents the example of the Delaunay graph applied to the faces of the primal graph.

Generally speaking, the model of Delaunay graph can be applied not only to the entities of the primal graph but to any element of the urban environment with the geo-referencing propose. For instance, we can consider in a natural way a Delaunay graph where the nodes are the buildings that form the urban building layer.
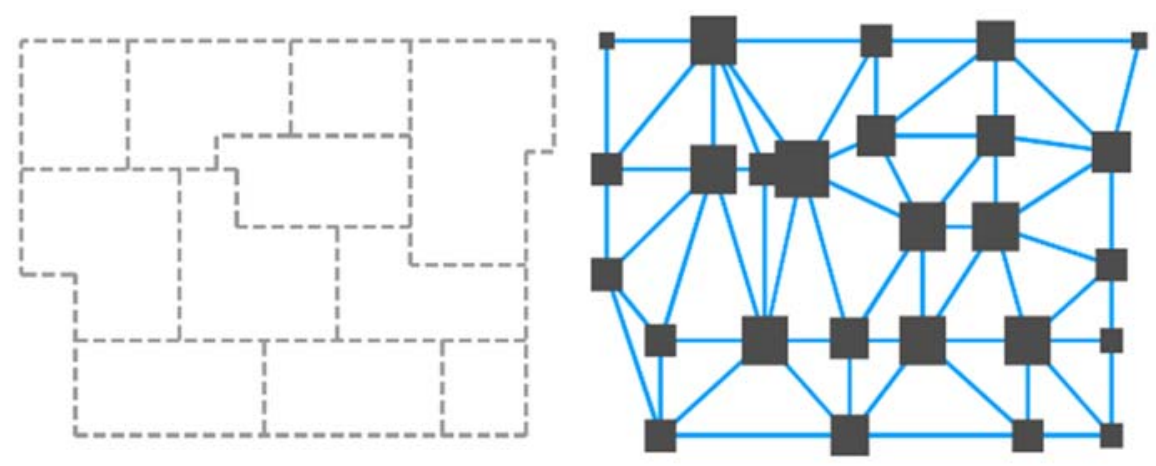

Figure 6: Constrained Delaunay graph of an abstract urban space. 


\section{COMPARING DIFFERENT MODELS FOR SPATIAL NETWORKS; A CASE STUDY, ALICANTE}

In the previous sections we have seen different types of graphs to represent the urban network. In summary, we can list different types of graphs associated with urban networks, such as the following:

- $\quad$ Primal graph: The nodes are street intersections and the edges are the streets.

- Edges to vertex dual graph: the nodes are the streets and the edges are the street intersections.

- Edge to vertex dual graph using the street names as edges continuity criteria.

- Edge to vertex dual graph using the linearity of streets as edges continuity criteria.

- Face to vertex dual graph: the nodes are primal graph faces and the edges are the edges of the primal graph.

- Delaunay graph.

In order to compare the key aspects of the previously mentioned graphs, we will implement the most representative of them to a case study as it is the urban network of Alicante, Spain. Since we take the city of Alicante as a reference in this work, we briefly expose some characteristics of it to understand the urban environment under study.

Alicante is a city of metropolitan character, located on the coast of the southeast of the country. It is a city with a heterogeneous urban morphology, which combines different urban patterns throughout its historical evolution. The historical part of the city is characterized by its irregular layout of the streets with significant formal discontinuities; in addition, this part of the city stands out for the remnants of walls and the castle, which can be seen from almost all parts of the city.

This set of heterogeneities of urban morphology has served as a motivation to implement the analysis of its characteristics from the point of view of the complex network theory.

This analysis is focused on the area of 4 square kilometers of the city, which includes both the historical part, and some new important extensions. In Fig. 7 it is shown the urban layout of the studied area of Alicante and a set of graphs that represent the urban network of the studied area.

Fig. 8 displays the degree distribution of the graphs in Fig. 7, while Table 1 summarizes the basic characteristics of the implemented graphs.

From Fig. 7(a) we can see that the primal graph of the street network of Alicante is a sparse and uniform graph, according to a very low degree distribution $P(k)$ where the values of the node's degree $k$ range only from 2 to 5 , as it can be seen in Fig. 8(a). These characteristics of the graph makes difficult to extract any consistent conclusions about the morphology of the urban network. The characteristics of edge to vertex dual graph, represented in Fig. 7(b), also cannot provide us enough information about the topology of the urban network. The degree distribution of this graph remains fairly uniform, even though the values of node's degree cover a slightly larger range, as it is shown in Fig. 8(b). It is worth noting that, despite having different number of nodes, the diameter of this graph is same as of that of the primal graph.

The characteristics of the urban morphology become more evident when we use the same edge to vertex dual graph but applying the criteria of street linearity, see Fig. 7(c). 

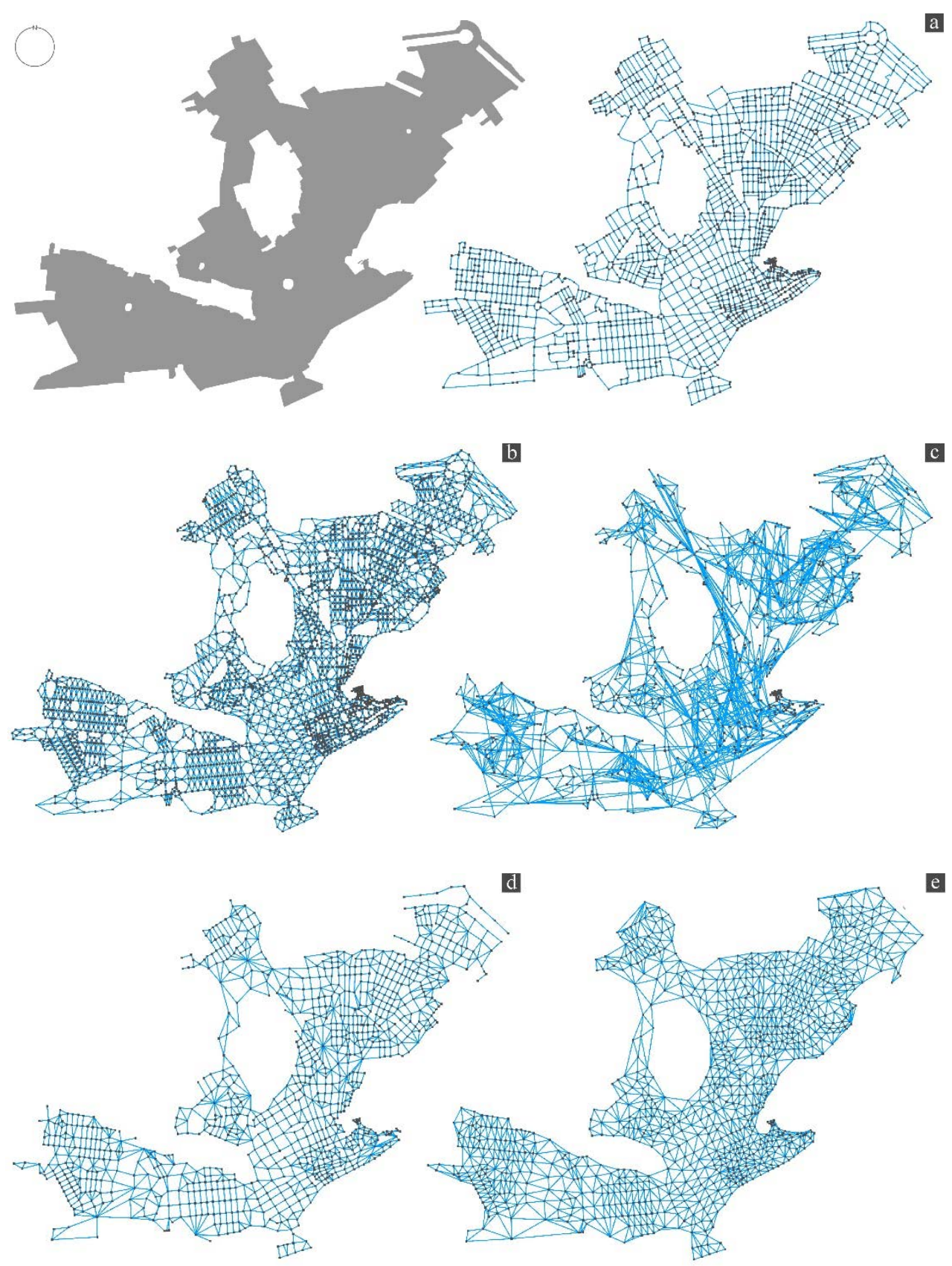

Figure 7: Different graph types of the urban network of Alicante; (a) Primal graph; (b) Edge to vertex dual graph; (c) Edge to vertex dual graph (street linearity criteria); (d) Face to vertex dual graph; (e) Delaunay graph by faces. 

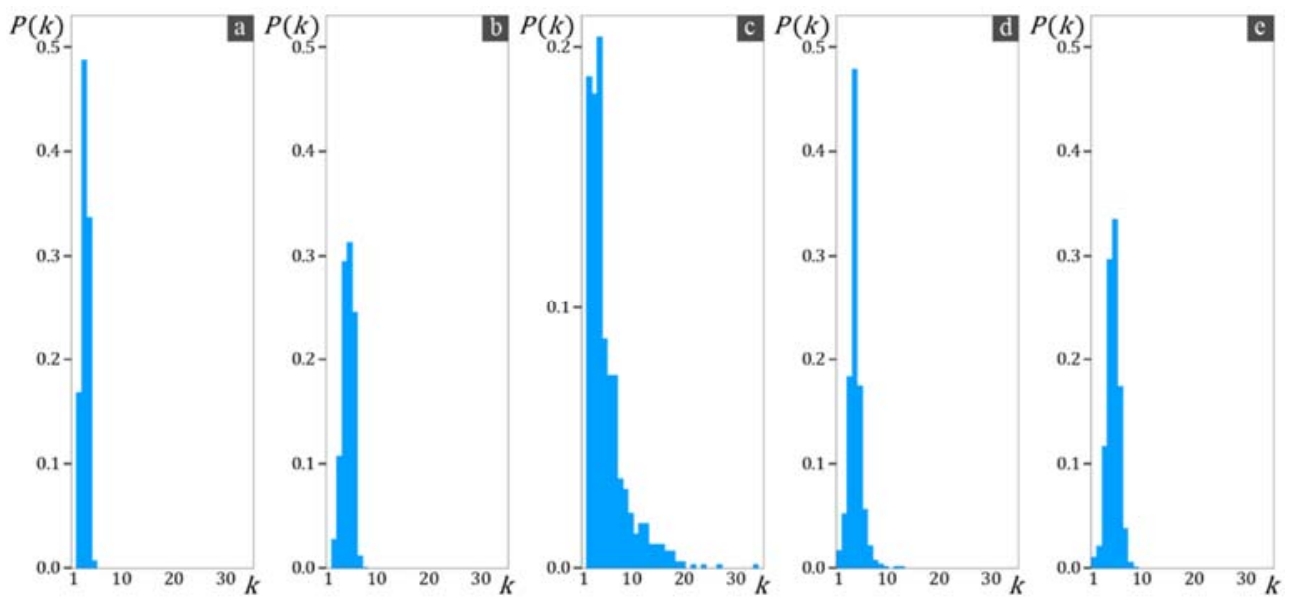

Figure 8: Degree distribution of the graphs.

Table 1: Characteristics of graphs that represent the spatial network of Alicante.

\begin{tabular}{|l|l|l|l|l|l|}
\hline \multirow{2}{*}{ Graph types } & \multicolumn{6}{|l|}{ Graph properties } \\
\cline { 2 - 6 } & Nodes & Edges & $\begin{array}{l}\text { Graph } \\
\text { diameter }\end{array}$ & $\begin{array}{l}\text { Graph } \\
\text { density }\end{array}$ & $\begin{array}{l}\text { Average } \\
\text { degree }\end{array}$ \\
\hline Primal graph & 1802 & 2861 & 96 & 0.0018 & 3.18 \\
\hline Edge to vertex dual graph & 2861 & 6690 & 96 & 0.0016 & 4.68 \\
\hline $\begin{array}{l}\text { Edge to vertex dual graph } \\
\text { (linearity of street criteria) }\end{array}$ & 758 & 4084 & 20 & 0.0142 & 10.77 \\
\hline Face to vertex dual graph & 1057 & 2169 & 71 & 0.0039 & 4.10 \\
\hline Delaunay graph & 1057 & 2978 & 55 & 0.0053 & 5.63 \\
\hline
\end{tabular}

Remark that the density is higher and its diameter is reduced. On the other hand, the degree distribution is no longer uniform, while the average degree it is considerably increased, as we can appreciate from Fig. 8(c) and Table 1. In addition, this degree distribution follows a function with negative slope that decreases exponentially, which is a typical characteristic of scale-free networks. The disposition of primal graph faces is studied through the face to vertex dual and Delaunay graphs, represented respectively in Fig. 7(d) and 7(e). Both graphs have close to normal degree distribution with slight tendency to the left in the case of the face to vertex dual graph, see Fig. 8(d) and 8(e).

\section{CONCLUSIONS}

In this work, we have shown different types of graphs to represent the urban network, as well as their most important characteristics. We have compared characteristics of the graphs through a case study of the urban network of Alicante. It has been verified that the primal graph is suitable to represent the topology and geometry of the urban layout but its low degree distribution does not allow us to identify characteristics of the network morphology. In order to identify the morphological characteristics of the urban layout we have to study its edge to vertex dual graph and take the additional criteria providing the individual graph edges with a spatial meaning. Also, we have shown how the regions or not transited urban 
space can be represented by the faces of the primal graph. Then, we can study the disposition and topological relationships of that faces by using the face to vertex dual graph or their spatial relationships by using the Delaunay graph.

\section{ACKNOWLEDGEMENT}

This work was partially supported by the Spanish Government, Ministerio de Economía y Competividad, which reference number is TIN2014-53855-P.

\section{REFERENCES}

[1] Delgado, M., El Espacio Público Como Ideología, La Catarata: Madrid, 2011.

[2] Castells, M., The Informational City: Economic Restructuring and Urban Development, Wiley-Blackwell Publishers: Chichester, Weinheim and Frederiksberg, 1991.

[3] Jacobs, J., The Depth and Life of Great American Cities, Random House: New York, 1961.

[4] Jacobs, J., Cities and the Wealth of Nations, Viking: Indiana, 1985.

[5] Townsend, A., Smart Cities: Big Data, Civic Hackers, and the Quest for a New Utopia, WW Norton \& Co: New York, 2013.

[6] Batty, M., Big Data, Smart Cities and City Planning. Dialogues in Human Geography, 3(3), pp. 274-179, 2013.

[7] Kitchin, R., The Real-Time City? Big Data and Smart Urbanism. GeoJournal, 79(1), pp. 1-14, 2014.

[8] Blanchard, P. \& Volchenkov, D., Mathematical Analysis of Urban Spatial Networks, Springer: Berlin, 2009.

[9] Barthélemy, M., Spatial Networks. Physics Report, 499(1), pp. 1-101, 2011.

[10] Okabe, A. \& Kokichi, S., Spatial Analysis along Networks. Statistical and Computational Methods, John Willey \& Songs: West Sussex, 2012.

[11] Friedkin, N., Theoretical foundations for centrality measures. American Journal of Sociology, 96(1), pp. 1478-1504, 1991.

[12] Freeman, L.C., Centrality in Social Networks: Conceptual Classification. Social Networks, 1(3), pp. 215-239, 1979.

[13] Freeman, L.C., A Set of Measures of Centrality Based on Betweenness. Social Networks, 40(1), pp. 35-41, 1977.

[14] Bonacich, P., Power and Centrality: A Family of Measures. American Journal of Sociology, 92(1), pp. 1170-1182, 1987.

[15] Borgatti, S.P., Centrality and Network Flow. Social Networks, 27(1), pp. 55-71, 2005.

[16] Crucitti, P., Latora, V. \& Porta, S., The Network Analysis of Urban Streets: A Primal Approach. Environment and Planning B, 33(5), pp. 705-725, 2006.

[17] Barabási, A.L., Linked: The New Science of Networks, AAPT: New York, 2003.

[18] Watts, D.J. \& Stroatz, S., Collective Dynamics of Small-Worlds Networks. Nature, 393(6684), pp. 440-442, 1998.

[19] Crucitti, P., Latora, V., Porta, S., The Network Analysis of Urban Streets: A Dual Approach. Physics A: Statistical Mechanics and its Applications, 369(2), pp. 853866, 2006.

[20] Harary, F. \& Norman, R.Z., Some Properties of Line Digraphs. Rendiconti del Circolo Matematico di Palermo, 9(2), pp. 161-169, 1960. 
82 Computational Methods and Experimental Measurements XVIII

[21] Jiang, B. \& Claramunt, C., Topological Analysis of Urban Street Networks. Environment and Planning B, 31(1), pp. 151-162, 2004.

[22] Jiang, B., Topological Analysis of Urban Street Networks: Universality and Peculiarity. Physica A: Statistical Mechanics and its Applications, 384(2), pp. 647655, 2004.

[23] Köhler, W., Gestalt Psychology: An Introduction to New Concepts in Modern Psychology, Liveright: New York, 1992.

[24] Thomson, R.C., Bending the Axial Line: Smoothly Continuous Road Centre-Line Segments as a Basis for Road Network Analysis. Proceedings of the Fourth International Space Syntax Symposium, London, 2003.

[25] Hiller, B., The Architecture of the Urban Object. Ekistics, 334(1), pp. 5-21, 1989.

[26] Hiller, B., The Social Logic of Space, Cambridge University Press: Cambridge, 2003.

[27] Whitney, H., Non-Separable and Planar Graphs. Transactions of the American Mathematical Society, 34(1), pp. 339-362, 1932.

[28] Yang, J., Estivil-Castro, V., Chalup, S.K., Support Vector Clustering through Proximity Graph Modelling. Proceedings of the Ninth International Conference on Neural Information, IEEE, pp. 898-903, 2002.

[29] Chew, L.P., Constrained Delaunay Triangulations. Proceedings of the Third Annual Symposium on Computational Geometry, ACM, pp. 215-222, 1987.

[30] Gabriel, K.R. \& Sokal, R.R., A New Statistical Approach to Geographic Variation Analysis. Society of Systematic Biologists, 18(3), pp. 259-270, 1969. 